\author{
Dany Bouchard \\ École de bibliothéconomie et des sciences de l'information \\ Université de Montréal
}

\title{
Dimensions organisationnelles des dépôts institutionnels : une approche sociotechnique
}

Résumé : Cet article présente brièvement notre projet de recherche doctorale. À travers le rôle et les fonctions des bibliothèques universitaires, nous cherchons à comprendre les facteurs sociaux, économiques, institutionnels et documentaires ayant un impact sur l'intégration des dépôts institutionnels dans l’infrastructure informationnelle des universités canadiennes.

\begin{abstract}
This paper briefly presents our doctoral project. Throughout the role and the responsibilities of academic libraries, we aim to understand the social, economical, institutional and documentary factors influencing the integration of institutional repositories into the information infrastructure of Canadian universities.
\end{abstract}

\section{Introduction}

Cet article présente brièvement notre projet de recherche doctorale. À travers le rôle et les fonctions des bibliothèques universitaires, nous cherchons à comprendre les facteurs sociaux, économiques, institutionnels et documentaires ayant un impact sur l'intégration des dépôts institutionnels dans l'infrastructure informationnelle des universités canadiennes. Dans la première section de ce texte, nous précisons brièvement le concept de dépôt institutionnel et son contexte, notre problématique de recherche, ainsi que les principaux objectifs qui en découlent. Dans la seconde partie, nous présentons le domaine de la socio-informatique, l'approche sociotechnique que nous utiliserons pour étudier ce nouveau phénomène, ainsi que le concept d'utilisabilité organisationnelle qui nous servira de cadre conceptuel. La troisième section concerne les aspects méthodologiques de notre démarche : la stratégie de recherche, les modes de collecte et d'analyse des données et les questions relatives à la qualité de la recherche. Finalement, nous terminons cette présentation par quelques réflexions sur les contributions théoriques, méthodologiques et pratiques espérées dans le cadre de ce projet de recherche.

\section{Contexte et problématiques}

« La mission du libre accès, dont nous avons rappelé qu'elle fondait le modèle bibliothéconomique dans son articulation avec l'édition, est aujourd'hui remplie de plus en plus efficacement dans le monde scientifique par d'autres systèmes que la bibliothèque traditionnelle, même si parfois cette dernière en reste un acteur »

(Salaün 2004).

Comme de nombreux acteurs de la chaîne documentaire, la bibliothèque universitaire a été et est encore profondément bouleversée par l'avènement du document numérique. Traditionnellement l'intermédiaire le plus proche du lecteur (Chartron 2002), elle vit à l'heure du numérique de profondes transformations qui vont jusqu'à remettre en cause sa mission fondamentale : mettre à la disposition de la communauté qu'elle dessert de l'information qui soit fiable et de qualité (Campbell 2006; Dempsey 2006; Salaün 2004). Cette nouvelle réalité, conséquence des transformations apportées par le document numérique et par les technologies de l'information et de la communication (TIC) sur les 
modes de diffusion et d'accès à l'information scientifique, oblige la bibliothèque universitaire à repenser en profondeur son offre de services (Budd 2005; Campbell 2006; Dempsey 2006; Lavoie et al. 2006; Salaün 2004; Salaün 2006). En 2005, 40 \% des universités américaines ayant un programme de doctorat avaient implanté un dépôt institutionnel et plus de $88 \%$ des autres prévoyaient en implanter un dans les six prochains mois. Dans la très grande majorité des cas, les bibliothèques assument le leadership en ce qui concerne l'implantation et l'administration de ce nouveau type de systèmes d'information documentaire (Lynch \& Lippincott 2005).

\section{$2.1 \quad$ Nature et fonctions des dépôts institutionnels}

Il existe plusieurs définitions de ce qu'est, ou de ce que devrait être, un dépôt institutionnel (Bailey 2005; Suber 2005). La définition de la Scholarly Publishing and Academic Resources Coalition (SPARC) formulé par (Crow 2002) est sans doute la plus généralement acceptée : Un dépôt institutionnel est une « collection numérique capturant et préservant le capital intellectuel d'une ou de plusieurs institutions universitaires » (Crow 2002). Cette collection possède quatre caractéristiques essentielles qui la définit par rapport à d'autres. Elle est institutionnelle et à teneur académique. Elle doit également être cumulative et perpétuelle. Enfin, les dépôts (et la collection) doivent être interopérables entre eux et ouverts aux membres de la communauté scientifique (Crow 2002). Mais le concept est nouveau et encore mal défini. Compte tenu du contexte économique et politique dans lesquels il s'inscrit, il suscite de nombreux débats où s'affrontent différentes conceptions relativement à sa nature, ses fonctions et la teneur des contenus qui y sont entreposés. Pour les membres de la communauté scientifique qui ont développé le libre accès, les dépôts institutionnels sont des outils permettant d'augmenter la visibilité et l'impact des travaux scientifiques (Swan et al. 2005). Pour les bibliothèques, il s'agit plutôt d'apporter une réponse valable aux problèmes posés par le numérique et plus particulièrement par l'augmentation des coûts d'abonnement aux périodiques (Chartron \& Salaün 2000). Pour le SPARC, les dépôts institutionnels offrent une alternative viable au système de publication scientifique actuel (Crow 2002). Enfin, pour les universités, ils représentent une opportunité d'assurer la pérennité de leur production et leur crédibilité par la qualité des travaux qu'elles commanditent (Crows 2002).

Pour une bibliothèque, l'implantation, l'administration et la promotion des dépôts institutionnels posent des défis importants. Ces activités exigent en effet une transformation majeure de la mission de la bibliothèque qui s'oriente désormais vers la préservation et la diffusion du capital intellectuel de l'institution (Salaün 2006). Cette nouvelle mission implique également un renversement du mode d'opération traditionnel de la bibliothèque qui était de rassembler l'information scientifique en fonction des besoins des membres de sa communauté (de l'extérieur vers l'intérieur) et qui est maintenant de diffuser le capital intellectuel de l'institution vers l'ensemble de la communauté scientifique (de l'intérieur vers l'extérieur) (Salaün 2006). Enfin, de par la nature et la diversité des documents qui y sont déposés, en fonction des possibilités offertes par le numérique et surtout parce qu'ils sont construits sur le principe de l'autoarchivage, les dépôts institutionnels ont également un impact déterminant sur tout le processus de traitement documentaire et, par conséquent, sur le rôle et la fonction des bibliothèques et des bibliothécaires.

Or, on connaît très peu les conséquences sociales, économiques et culturelles liées l'implantation de ce type de systèmes pour les bibliothèques universitaires. La grande majorité des travaux dans ce domaine s'intéressent plutôt aux raisons de la non-utilisation 
des dépôts par les membres de la communauté (Allen 2005; Foster \& Gibbons 2005; Sparks 2005) ou encore à l'impact du libre accès (le facteur d'impact) sur l'accès aux résultats de la recherche (Hitchcock 2006). Il existe également de nombreuses descriptions applicatives ${ }^{1}$ (application description) sur les expériences d'implantation des dépôts dans les bibliothèques, ce type d'études ayant comme principal objectif de décrire l'expérience des auteurs lors l'implantation d'un système d'information spécifique dans un contexte spécifique. Cependant, ce ne sont pas des recherches empiriques au sens strict ayant comme objectif la compréhension profonde d'un phénomène (Benbasat et al. 1987).

\subsection{But, objectifs et questions de recherche}

Bien que de nombreuses recherches dans les domaines des systèmes d'information et des bibliothèques numériques aient démontré que l'adoption et l'usage des systèmes documentaires varient en fonction du contexte d'utilisation (Bishop \& Star 1996; Covi \& Kling 1996; Elliott \& Kling 1997; Kling \& Lamb 1999; Orlikowski 1992; Walsham 1993), il n'existe pas de recherche empirique dont le but est de comprendre comment les dépôts institutionnels s'intègrent dans le réseau informationnel des universités et des bibliothèques de recherche. Dans ce contexte, nos principaux objectifs sont de :

- Modéliser le concept de dépôts institutionnels d'un point de vue sociotechnique;

- Déterminer le degré d'adéquation (utilisabilité organisationnelle) entre les caractéristiques sociotechniques des dépôts institutionnels et les dimensions organisationnelles dans lesquelles ils s'inscrivent;

- Proposer des stratégies permettant d'assurer l'intégration des dépôts institutionnels dans les bibliothèques universitaires.

Pour atteindre ces différents objectifs, nous nous proposons de répondre aux questions de recherche suivantes :

1. Quels sont les principaux acteurs impliqués dans le processus d'implantation et de gestion des dépôts institutionnels ? Et comment ces différents acteurs perçoiventils les dépôts institutionnels ?

2. Quelles forces sociales, économiques et politiques gouvernent et influencent l’intégration et le développement des dépôts institutionnels?

3. Comment, d'un point de vue organisationnel, peut-on assurer l'utilisabilité des dépôts institutionnels?

\section{Approche théorique et cadre analytique de la recherche}

La majorité des recherches dans le domaine des systèmes d'information conçoivent les TIC uniquement comme des outils permettant ou facilitant la réalisation d'une tâche (Kling \& Lamb, 1999; Kling \& al., 2000b). La métaphore de l'outil est utile (et très répandue) car elle permet d'envisager l'usage immédiat le plus approprié pour une technologie (Kling \& al., 2000). Dans cette section, nous présentons brièvement la socioinformatique, un domaine de recherche qui réfute ce modèle et qui propose une vision sociotechnique des technologies de l'information et de la communication (TIC). Nous discuterons aussi le concept d'utilisabilité organisationnelle, un cadre d'analyse issu des recherches dans ce domaine qui formalise l'étude des TIC dans une perspective institutionnelle. 


\title{
3.1 Une approche sociotechnique : la socio-informatique
}

La socio-informatique se caractérise par la nature des sujets étudiés (problèmes qui surgissent des interrelations entre le contexte social, la conception, l'implantation et l'utilisation des TIC) plutôt que par les théories ou les méthodes utilisées pour aborder ces différentes questions (Kling et al. 2000; Sawyer \& Eschenfelder 2002). Cette discipline se définit comme "l'étude multidisciplinaire du design, de l'utilisation et de l'impact des technologies de l'information qui tient compte des interactions avec les contextes culturels et organisationnels dans lesquels ces technologies existent » (Lamb \& Sawyer 2005). Elle est née de la volonté de chercheurs de disciplines variées (informatique, bibliothéconomie et sciences de l'information, sociologie des sciences, management des systèmes d'information) de rassembler sous un même domaine les recherches s'intéressant aux aspects sociaux des TIC (Kling 2000).

Notre démarche s'inscrit dans ce paradigme et s'appuie sur deux de ses idées maîtresses. D’abord, l'idée que les technologies doivent être conceptualisées comme socialement construites, ce qui implique qu'elles sont dans un processus de construction réciproque avec le contexte et qu'elles ne peuvent être comprises (ni étudiées) sans faire référence à celui-ci (Eschenfelder \& Chase 2002).

\begin{abstract}
The definition of social informatics helps to emphasize a key idea: ICTs do not exist in social or technological isolation. Their cultural and institutional contexts influence the ways in which they are developed, the kinds of workable configurations that are proposed, how they are implemented and used, and the range of consequences that occur for organizations and other social groupings (Kling 2000 : 15).
\end{abstract}

Ce concept est capital dans notre démarche. Il suggère que des systèmes similaires peuvent avoir des conséquences différentes dans différents contextes sociaux ou institutionnels. Comme le souligne Rosenbaum (2004 : 11) : "There are appeals to the concept to explain why the same software can be implemented in similar organisations and the result in different and sometimes contradictory outcomes”.

La deuxième idée issue de ce domaine découle de la première en ce sens qu'elle conceptualise les technologies comme un réseau d'interactions sociotechniques (RIST) (Kling \& Lamb 1999; Kling et al. 2000. Kling et al. 2003)

Le RIST inclut des personnes (et les organisations), de l'équipement, diverses ressources (argent, habileté, statut), des documents et des messages, des arrangements légaux et des mécanismes de renforcement ainsi que des flux de ressources. Les éléments [nœuds] du RIST sont hétérogènes. Le réseau d'interrelations entre ces éléments est de natures sociales, économiques et politiques (Kling \& al., 2003 : 48 - notre traduction).

D'un point de vue organisationnel et conceptuel, cette approche des TIC a des conséquences analytiques importantes par rapport au modèle traditionnel de l'outil. C'est une approche holistique à l'étude des systèmes d'information qui permet une analyse en profondeur des transformations subies par les organisations dans un environnement numérique (Kling \& Lamb 1999). Le tableau suivant présente quelques-unes des différences entre les deux approches. 
Tableau 1 : Caractéristiques des approches outils et systèmes sociotechniques

\begin{tabular}{|l|l|}
\hline MODÈLE STANDARD (OUTIL) & MODÈLE SOCIOTECHNIQUE \\
\hline - Les TIC sont des outils & - Les TIC sont des réseaux sociotechniques \\
- L’implantation n'est pas un processus (one & - L’implantation est un processus dans le temps \\
shot implanmentation) & - L'effet des TIC est indirect et implique différentes \\
- Les effets technologiques sont directs et & échelles temporelles \\
immédiats & - Les aspects politiques sont fondamentaux \\
- Les aspects politiques ne sont pas importants & - Le contexte est complexe \\
- La contexte est simple & - La connaissance et l'expertise sont \\
& fondamentalement tacites/implicites
\end{tabular}

Source : adapté de Kling \& Lamb, 1999 : 19 - notre traduction

C'est en nous appuyant sur ces deux construits de la socio-informatique que nous modéliserons les dépôts institutionnels comme des systèmes essentiellement sociotechniques. Après avoir développé un premier modèle théorique, nous pourrons l'enrichir à partir des données empiriques recueillies afin d'explorer le processus d'intégration des dépôts institutionnels dans les pratiques organisationnelles des bibliothèques universitaires.

\subsection{L'utilisabilité organisationnelle des dépôts institutionnels}

Depuis plus d'une décennie, de plus en plus de chercheurs en informatique sociale s'intéressent aux aspects sociaux des bibliothèques numériques (Social Informatics of Digital Libraries) (Bishop et al. 2003; Bishop et al. 2000; Bishop \& Star 1996; Borgman 1997; Borgman 2003; Davies 1997). Bishop \& Starr (1996) ont identifié et documenté deux principaux axes de recherche dans ce champ disciplinaire qu'elles définissent comme "the study of social influences, processes, practices, and effects related to how knowledge is structured and communicated in DLs" (Bishop \& Starr 1996 : 302). Le premier de ces axes, la manière dont l'information est structurée dans les bibliothèques numériques, concerne le rôle du catalogue et du document dans un environnement digital, la relation entre documents et liens, la construction sociale des technologies et les interrelations entre documents et activités sociales. Le second axe s'intéresse à l'utilisation des bibliothèques numériques, à la nature des interactions sociales lors de cette utilisation ainsi qu'aux aspects organisationnels liés à l'implantation et à l'utilisation de ces systèmes. Cette catégorisation des recherches dans le domaine est plus conceptuelle et analytique que pratique. Plusieurs recherches les combinant afin de permettre une compréhension plus fine des enjeux liés aux dimensions sociales des bibliothèques numériques. Dans le cadre de notre thèse, nous nous attarderons plus particulièrement aux facteurs organisationnels liés à l'implantation des dépôts institutionnels à travers le concept d'utilisabilité organisationnelle sans toutefois ignorer les autres dimensions possibles à l'étude des bibliothèques numériques et des dépôts en particulier.

Le concept d'utilisabilité organisationnelle (Elliott \& Kling 1996) a été pour la première fois défini sous le terme de validité organisationnelle par (Markus \& Robey 1983 : 203) comme : "The fit between an information system and its organizational context of use". Pour les auteurs, la validité organisationnelle n’est pas une propriété du système ni de l'organisation, mais bien de l'interrelation entre les deux. Elle se mesure en fonction des relations entre un système et différentes dimensions de l'organisation : les usagers, la structure organisationnelle, la distribution du pouvoir à l'intérieur de l'organisation et 
l'environnement. Bien qu'il soit utile pour évaluer l'intégration des systèmes d'information dans les organisations, le concept n'est pas absolu et normatif et reste descriptif. Un système pouvant être valide à un niveau d'analyse et invalide à un autre (Markus \& Robey 1983).

Kling \& Elliott (1994) et Elliott \& Kling (1997) ont par la suite enrichi le concept et ramené à trois (individuel, organisationnel et environnemental) les quatre niveaux d'analyse développés par Markus \& Robey (1983). À partir de données empiriques recueillies dans le cadre d'une recherche sur l'utilisabilité organisationnelle des bibliothèques numériques dans le domaine juridique, ils ont raffiné ces différents niveaux d'analyse en développant différentes dimensions d'intégration des bibliothèques numériques en fonction du contexte. Cette approche permet une analyse approfondie et plus précise des caractéristiques sociotechniques des bibliothèques numériques et, en ce qui nous concerne, des dépôts institutionnels.

Tableau 2 : Concept d'utilisabilité organisationnelle

\begin{tabular}{|l|l|}
\hline NIVEAU D’ANALYSE & DIMENSIONS ORGANISATIONNELLES \\
\hline \multirow{3}{*}{ Individuel } & $\begin{array}{l}\text { - Intégration dans les pratiques de travail } \\
\text { - Fiabilité de l'information; } \\
\text { - Acceptabilité sociale et culturelle }\end{array}$ \\
\hline \multirow{3}{*}{ Organisationnel } & $\begin{array}{l}\text { - Structure organisationnelle } \\
\text { - Distribution du pouvoir } \\
\text { - Normes institutionnelles } \\
\text { - Organisation sociale de l'infrastructure technologique }\end{array}$ \\
\hline \multirow{2}{*}{ Environnemental } & $\begin{array}{l}\text { - Influence de l'environnement sur les contenus } \\
\text { - Écologie de travail }\end{array}$ \\
\hline
\end{tabular}

Source : Elliott \& Kling, 1996 : 1027 - notre traduction

Enfin, Davis (1997) puis (Xie \& Wolfram 2002) ont utilisé le modèle pour étudier l'impact des dimensions organisationnelles sur le développement des bibliothèques numériques dans un contexte universitaire. Davies (1997) a modélisé les principaux acteurs impliqués dans le développement d'une bibliothèque universitaire numérique : les instigateurs et responsables de projet, le personnel de la bibliothèque, les développeurs d'application et les usagers. Les travaux de Xie et Wolfram (2002) ont, quant à eux, validé et raffiné ce modèle en incorporant trois modes d'interactions possible entre ces différents acteurs, soit l'influence des uns sur les autres, les communications entre eux et les activités ou services qu’ils s’offrent.

\subsection{Limites du modèle}

Ce modèle nous semble particulièrement adapté à notre problématique et à nos objectifs de recherche. Cependant, nous croyons qu'il pourrait être ajusté afin d’intégrer les spécificités des dépôts institutionnels par rapport aux bibliothèques numériques. Par exemple, il repose sur une conception de l'usager qui est difficilement applicable dans le contexte des dépôts institutionnels, puisque la nature ouverte des systèmes (libres accès et logiciels libres) implique un réajustement du concept même d'usager. Dans le contexte des dépôts, les usagers finaux du système sont aussi les fournisseurs de contenu. De la même façon, parce que la plupart des systèmes sont des logiciels libres, le personnel de la bibliothèque est amené à participer au développement du système ou des applications connexes. Des recherches dans le domaine de l'informatique sociale ont d'ailleurs déjà 
commencé à explorer cette piste et à développer un nouveau concept d'usager (l'acteur social) qui est mieux adapté à la nouvelle réalité numérique (Davenport \& al., 2003; Lamb \& Kling, 2003; Lamb, 2005; 2006). Enfin, le modèle tel qu'il est présenté ici ne tient pas assez compte des questions documentaires liées à l'impact social du document numérique sur les pratiques de travail dans les bibliothèques et dans les universités. La dimension environnementale aborde cet aspect, mais sans réellement la développer.

Il nous apparaît donc pertinent et nécessaire d'intégrer le concept d'acteur social et celui de réseau d'interaction sociotechnique (RIST) au concept d'utilisabilité organisationnelle afin de développer un cadre conceptuel qui soit mieux adapté aux caractéristiques spécifiques des dépôts. Ce nouveau modèle permettrait en outre de rendre compte de l'impact du document numérique sur les processus documentaires des bibliothèques et des bibliothécaires dans un environnement numérique et en réseau. L’intégration de ces différents concepts fera donc l'objet d'un développement important dans le cadre de notre proposition de recherche.

\section{Méthodologie}

Les questions d'ordre méthodologique sont intimement liées à la position épistémologique du chercheur et de celle-ci découlent directement les choix relatifs à la conception de la recherche, à l'opérationnalisation de la collecte et à l'interprétation des données (Gohier 2004; Martineau 2005). Adoptant une approche sociotechnique, notre démarche s'inscrit dans le paradigme interprétatif de la recherche sur les systèmes d'information. Contrairement à l'approche positiviste qui postule une réalité objective dont la nature peut être découverte, caractérisée et mesurée empiriquement (Orlikowski \& Baroudi 1991), les recherches interprétatives affirment au contraire une réalité multiple et socialement construite (Klein \& Myers 1999; Orlikowski \& Baroudi 1991; Walsham 1995). L'objectif principal est alors «de comprendre [interpréter] le sens d'un phénomène à travers la signification que les individus [y compris le chercheur] lui assignent " (Klein \& Myers 1999: 69 - notre traduction). Plutôt que d'évacuer sa subjectivité, le chercheur la reconnaît et l'inclut dans le processus même de la recherche (Laperrière 1997). La qualité et la rigueur sont atteintes à travers différentes stratégies dont la triangulation qui « cherche plus à capturer les multiples dimensions de la réalité (sociale, culturelle, économique, etc.) plutôt qu’une vérité absolue » (Patton 2002 : 546). En fait, les études de cette nature ne visent pas la généralisation de catégories abstraites, mais la cohérence et la compréhension profonde des différentes dimensions d'un phénomène (Walsham 1995 dans Klein \& Myers 1999).

\subsection{Stratégie de recherche.}

Largement utilisée et reconnue dans les recherches sur les aspects sociaux des systèmes d'information (Klein \& Myers 1999; Orlikowski \& Baroudi 1991; Walsham 1993; 1995; 2006), l'étude de cas permet d'étudier un phénomène contemporain dans son contexte, surtout lorsque les frontières entre le phénomène et le contexte ne sont pas clairement définies (Yin 2003). L'utilisation de la méthode de cas est justifiée lorsque le phénomène qui est l'objet d'intérêt exige une approche holistique, une investigation en profondeur et qu'il doit être étudié dans son contexte naturel pour être compris (Benbasat \& al., 1987; Yin 2003). Nous avons opté pour une stratégie par cas multiples, car elle est la plus appropriée à notre démarche qui vise à comprendre l'intégration des dépôts dans les bibliothèques universitaires, à nos objectifs de recherche et à notre approche théorique qui affirme l'indissociabilité du contexte social et institutionnel d'avec les systèmes 
d'information. En effet, « l'étude de plusieurs cas vise généralement à fournir une riche description du contexte dans lequel les évènements se déroulent et à mettre à jour la structure profonde des comportements sociaux » (Light, 1979 dans Gagnon, 2005 : 43).

\subsection{Unité d'analyse, terrain et échantillonnage}

Il y deux niveaux d'analyse possibles à l'étude de l'utilisabilité organisationnelle des dépôts institutionnels : d'abord d'un point de vue conceptuel en fonction de la relation entre le concept de dépôt et celui de bibliothèque. Est-ce que les dépôts institutionnels sont adaptés au contexte des bibliothèques ? Ensuite, d'un point de vue strictement organisationnel, c'est-à-dire entre les caractéristiques sociotechniques du système utilisé et les dimensions organisationnelles de la bibliothèque. Est-ce que le système $X$ est adapté à la bibliothèque $Y$ ? Dans le cadre de notre recherche, le second niveau d'analyse doit démontrer la pertinence du premier. Nous cherchons à comprendre l'utilisabilité organisationnelle des dépôts institutionnels à modéliser les interactions sociales, économiques et politiques entre les différents acteurs du réseau. Pour reprendre la formule de Markus \& Robey (1983), notre unité d'analyse n'est pas le dépôt institutionnel ni la bibliothèque, mais bien le degré d'adéquation (the fit) entre les deux.

Les cas seront déterminés en fonction d'un échantillon de type non probabiliste à choix raisonné (Patton, 2002). Nous utiliserons également l'échantillonnage théorique qui est une version plus conceptuelle de l'échantillonnage par critères et qui permet la sélection des cas en fonction des concepts émergents (Patton 2002). Afin d'atteindre la réplication théorique (Benbasat \& al. 1987; Yin 2003), nous visons à recruter des cas hétérogènes et atypiques. L'échantillonnage se fera en fonction des caractéristiques des systèmes et des sites (bibliothèques canadiennes ayant implanté un dépôt, caractéristiques et structure de la bibliothèque, degré d'implication des départements, nombre de documents présents, etc.) afin de permettre l'analyse inter cas (Laperrière 1997). Enfin, à l'intérieur de chacun des sites, les répondants seront sélectionnés en fonction de critères qui restent à déterminer, mais qui seront basés sur le cadre conceptuel et sur la modélisation sociotechnique des systèmes. Dans ce contexte, nous viserons la saturation théorique, qui sera atteinte lorsqu'il y aura redondance des données auprès des répondants (Fortin 1996 : 152).

\section{Modes de collecte}

Yin (2003 : 85) identifie six sources d'évidences possibles dans une étude de cas : la documentation, les archives, les entrevues, les observations directes, les observations participantes et les artefacts physiques. Nous utiliserons principalement les entrevues et l'analyse des systèmes comme principaux modes de collecte de données. Nous compléterons notre collecte par des observations directes dans les différents milieux étudiés et par l'analyse fine de la documentation et des archives disponibles.

\subsection{Analyse et modélisation des systèmes}

Cette première étape permettra de modéliser les systèmes en fonction du contexte. Elle sera effectuée en plusieurs phases. D’abord, nous réaliserons un modèle sociotechnique des dépôts institutionnels à partir de la littérature du domaine et en fonction de l'approche théorique utilisée. Ensuite, nous ajusterons le modèle théorique en fonction des résultats de l'analyse pour chacun des cas étudiés afin de produire un modèle sociotechnique du processus d'intégration des dépôts institutionnels qui soit ancré dans les données (Miles \& Huberman 2003; Strauss \& Corbin 2000). 


\subsection{Entrevue semi-structurée}

Les entrevues sont une des sources de données les plus importantes dans une étude de cas (Benbasat \& al. 1987; Yin 2003) et plus encore dans les études interprétatives où l'objectif est d'interpréter la réalité en fonction de la perception des individus (Klein \& Myers 1999; Walsham 1995; 2006). Nous prévoyons faire des entrevues avec les acteurs identifiés lors de la modélisation sociotechnique du système, notamment les responsables du projet et les bibliothécaires impliqués à un degré ou un autre dans les processus d'implantation, d'administration ou de promotion des dépôts. Elles nous permettront également d'explorer en profondeur les dimensions sociotechniques du concept d'utilisabilité organisationnelle puisque le questionnaire sera développé en fonction de notre approche théorique et de la modélisation des systèmes.

\subsection{Analyse de documents et observation directe}

Enfin, nous utiliserons l'observation directe et l'analyse de document comme sources de données complémentaires et contextuelles. L'analyse de la documentation nous permettra de valider les données obtenues par l'analyse des systèmes et par les entrevues (Yin 2003). Dans cet objectif, nous utiliserons plusieurs types de document: documents administratifs, documentation du système, mémos internes, politiques et procédures, statistiques d'utilisation, outils de promotion, etc. L'observation directe a comme objectif « la compilation de l'information la plus complète possible sur une situation sociale particulière » (Laperrière 1995: 253). Elle nous permettra d'appréhender le contexte social et culturel de l'organisation ainsi que les différentes interactions entre les intervenants et le système. L'observation sur le terrain permet en effet au chercheur de « s’immerger dans la situation sous étude » (Patton 2002 : 23 - notre traduction).

Le but de la triangulation des modes de collecte étant d'obtenir des données riches sur l'objet d'étude et d'en capturer toute la complexité contextuelle (Benbasat \& al. 1987 : 374). L'utilisation conjuguée de l'entrevue, de l'observation et de la documentation permet d'appréhender un phénomène sous ses multiples perspectives. La triangulation permettra une compréhension holistique du contexte des bibliothèques étudiées tel que conceptualisé dans les approches sociotechniques et dans notre cadre conceptuel. Chacune des méthodes ayant ses avantages et ses inconvénients, ses forces et ses faiblesses, la triangulation des méthodes de collectes nous permettra d'appréhender les multiples dimensions du contexte social et culturel d'une organisation (Patton 2002).

\section{Analyse des données}

Nous avons choisi comme méthode d'analyse l'approche développée par Mille \& Huberman (2003) qui, par bien des aspects, se rapproche de la théorisation ancrée développée par Glaser \& Strauss (1967). Elle est de nature inductive et s’appuie sur un processus itératif de comparaisons constantes entre la collecte et l'analyse, ce qui favorise l'émergence des concepts et des catégories en fonction des résultats de l'analyse (Laperrière 1997; Paillé 1994; Strauss \& Corbin 2000). Elle s'appuie également sur l'échantillonnage théorique comme méthode de sélection des cas (Laperrière 1997; Strauss \& Corbin 2000). Mais contrairement à la théorisation ancrée pure, l'approche analytique développée par Miles \& Huberman (2003) ouvre la recherche à plusieurs sites, ce qui implique la conception a priori d'outils de recherche (Laperrière 1997). Par conséquent, elle encourage le recours à la théorie lors des étapes conceptuelles et opératoires de la recherche (Laperrière 1997; Miles \& Huberman 2003) rejoignant en cela les chercheurs interprétivistes dans le domaine des systèmes d'information (Klein \& 
Myers 1999; Orlikwski 1992; Walsham 1995; 2006). En effet, dans les études de cette nature, la théorie peut être utilisée comme guide lors des étapes de design et de collecte, lors d'un processus itératif de collecte et d'analyse, comme produit final de la recherche ou lors de l'une ou l'autre de ces étapes, le chercheur devant rester souple et ouvert face aux données et prêt à modifier ses positions initiales en fonction du terrain (Walsham 1995; 2006).

\section{Opérationnalisation des critères de qualité}

Les critères de qualité (validité interne, validité externe, réplicabilité et objectivité) issus de la recherche quantitative et de la tradition positiviste qui visent une explication objective et décontextualisée d’un phénomène ainsi que la généralisation statistique des résultats ne peuvent s’appliquer aux études interprétatives (Laperrière 1997; Lincoln \& Guba 1985). Les critères constructivistes de crédibilité, de transférabilité, de stabilité et de confirmabilité développés par Lincoln \& Guba (1985) et largement utilisés dans les recherches qualitatives en sciences humaines et sociales sont plus adaptés à notre approche théorique et méthodologique.

Le critère de crédibilité concerne la valeur des données et des résultats obtenus en fonction des différentes réalités interprétées. Le critère de transférabilité diffère considérablement de celui de validité externe puisque les études interprétatives, il est impossible d'inférer les résultats en fonction d'une population (Lincoln \& Guba, 1985). On vise plutôt la transférabilité analytique (théorique) des résultats de la recherche (Yin 2003). La stabilité permet de démontrer que le processus de recherche est rigoureux et vérifiable et que les données obtenues peuvent résister à l'épreuve du temps (Guba \& Lincoln 1985). Enfin, la confirmabilité vise à s’assurer que les résultats obtenus reflètent bien les données ainsi que les conditions de la recherche et non le point de vue du chercheur qui doit faire preuve de transparence et affirmer ses biais (Lincoln \& Guba 1985). Différentes stratégies permettent d'assurer le respect de ces différents critères (Laperrière 1997; Lincoln \& Guba 1985) : prise en compte de la subjectivité, conception d'un protocole de recherche observation en contexte naturel, confrontation systématique des interprétations, codage précis et rigoureux, Triangulation des sources, des méthodes et des théories, , création d'un journal de bord, pour ne nommer que ceux-là. Conscients de l'importance déterminante de ces questions, nous tiendrons compte de l'ensemble de ces stratégies dans les différentes étapes de réalisation de notre projet afin d'assurer la qualité et la rigueur de celui-ci.

\section{Conclusion et contributions envisagées}

La question de l'accès à l'information scientifique comporte des enjeux socioéconomiques importants pour les bibliothèques universitaires. Les dépôts institutionnels représentent une stratégie intéressante dans ce contexte. Ils offrent aux bibliothèques, comme aux institutions universitaires, l'opportunité de se repositionner dans le nouvel environnement numérique (Aubry \& Janik 2005; Campbell 2006; Chartron 2002; Salaün 2004). Il est cependant important de bien comprendre les différents enjeux liés à l'intégration d'une nouvelle génération de systèmes documentaires dans les pratiques organisationnelles des bibliothèques et des bibliothécaires universitaires qui, déjà, sont profondément transformées par les spécificités du document numérique. C’est à ce niveau que se situe le principal intérêt de notre recherche. 
D’un point de vue pratique, ces travaux intéresseront les professionnels responsables de ces systèmes qui disposeront d'un cadre analytique leur permettant d'évaluer les spécificités sociotechniques des dépôts en fonction des caractéristiques propres aux universités canadiennes. D’un point de vue méthodologique, ils permettront de valider la méthode, les techniques et les outils développés pour étudier et comprendre cette nouvelle génération de systèmes d’information issus de l'évolution du Web 2.0. Enfin, d'un point de vue théorique, cette recherche permettra de valider l'importance du contexte institutionnel et culturel dans le processus d'implantation de systèmes d'information et de développer un modèle d'utilisabilité organisationnelle tenant compte des caractéristiques sociotechniques des systèmes, de la dimension sociale de l'usager ainsi que des processus liés au traitement documentaire des documents numériques. Finalement, elle permettra de modéliser le concept de dépôts d'un point de vue sociotechnique et, nous l'espérons, de comprendre les interactions sociales, économiques et politiques impliquées dans les processus liés à la communication scientifique, à l’enseignement et à la recherche.

\section{Bibliographie}

Allen, J. 2005. Interdisciplinary differences in attitudes towards deposit in institutional repositories. Master Interdisciplinary differences in attitudes towards deposit in institutional repositories, Manchester Metropolitan University.

Aubry, C. \& J. Janik. 2005. Les archives ouvertes : enjeux et pratiques, guide à l'usage des professionnels de l'information. ADBS-Édition.

Bailey, C.W. 2005. The role of reference librarians in institutional repositories. Reference Services Review 33: 259-67.

Benbasat, I., D.K. Goldstein, and M. Mead. 1987. The Case Research Strategy in Studies of Information-Systems. MIS Quaterly 11: 369-86.

Bishop, A.P., L.J. Neumann, S.L. Star, C. Merkel, E. Ignacio, and R.J. Sandusky. 2000. Digital libraries: Situating use in changing information infrastructure. Journal of the American Society for Information Science and Technology 51: 394-413.

Bishop, A.P. and S.L. Star. 1996. Social informatics of digital library use and infrastructure. Annual Review of Information Science and Technology 31: 301401.

Bishop, A.P., N.A. Van House, \& B.P. Buttenfield. 2003. Digital library use : Social practice in design and evaluation. Cambridge, MIT Press (Digital Libraries and Electronic Publishing).

Borgman, C.L. 1997. Workshop report: Social Aspects Of Digital Libraries. 2006. Available from http://www.dlib.org/dlib/january97/01clips.html [April 2007].

-----. 2003. The invisible library: paradox of the global information infrastructure. Library trends 51: 652-74.

Budd, J.M. 2005. The changing academic library operations, culture, environments. Chicago: Association of College and Research Libraries. 
Campbell, J.D. 2006. Changing a Cultural Icon: The Academic Library as a Virtual Destination. Available from http://www.educause.edu/ir/library/pdf/erm0610.pdf [April 2007].

Chartron, G. 2002. Les chercheurs et la documentation numérique : nouveaux services et usages. Paris, Cercle de la librairie (Collection Bibliothèques).

Chartron, G. and J.M. Salaün. 2000. La reconstruction de l'économie politique des publications scientifiques. Bulletin des bibliothèques de France 45: 32-42.

Covi, L.M. and R. Kling. 1996. Organizational dimensions of effective digital library use: closed rational and open natural systems models. Journal of the American Society for Information Science and Technology 47: 96-89.

Crow, R. 2002. The Case for Institutional Repositories: A SPARC Position Paper. ARL. Available from http://www.arl.org/sparc/bm doc/ir_final_release_102.pdf [April 2007].

Davies, D. 1997. Organizational influences on the university electronic library. Information Processing \& Management 33: 377-92.

Dempsey, L. 2006. The (Digital) Library Environment: Ten Years After. 2006. Available from http://www.ariadne.ac.uk/issue46/dempsey/ [April 2007].

Elliott, M.S. and R. Kling. 1997. Organizational usability of digital libraries: Case study of legal research in civil and criminal courts. Journal of the American Society for Information Science and Technology 48: 1023-35.

Eschenfelder, K.R. and L.C. Chase. 2002. Socio-Technical network of large, postimplementation web information systems : tracing effects and influences. Paper presented at 35th Hawaii International Conference on the System Sciences.

Foster, N.F. and S. Gibbons. 2005. Understanding Faculty to Improve Content Recruitment for Institutional Repositories. 2006. Available from http://dlib.org/dlib/january05/foster/01foster.html [April 2007].

Gagnon, Y.C. 2005. L'étude de cas comme méthode de recherche. Québec (Québec): Presses de l'Université du Québec.

Gohier, C. 2004. De la démarquation entre critères d'ordre scientifique et d'ordre éthique en recherche interprétative. 2006. Available from http://www.recherchequalitative.qc.ca/Textes/24gohier.pdf [April 2007].

Hitchcock, S. 2006. The effect of open access and downloads ('hits'), on citation impact : A bibliography of studies. The Open Citation Project - Reference Linking and Citation Analysis for Open Archives .

Klein, H.K. and M.D. Myers. 1999. A set of principles for conducting and evaluating interpretive field studies in information systems. MIS Quaterly 23: 67-93.

Kling, R., G. Mckim, J. Fortuna, and A. King. 2000. Scientific collaboratories as sociotechnical interaction network : A theorical approach. Paper presented at 
Proceedings of the Americas Conference on Information Systems, at Long Beach, CA.

Kling, R. 2000. Learning about information technologies and social change: The contribution of social informatics. Information Society 16: 217-32.

Kling, R. and R. Lamb. 1999. IT and organizational change in digital economies : a socio-technical approach. Computers and Society 29.

Kling, R., G. Mckim, and A. King. 2003. A Bit More to It: Scholarly Communication Forums as Socio-Technical Interaction Networks

4. Journal of the American Society for Information Science and Technology 54: 47-67.

Lamb, R. and S. Sawyer. 2005. On extending social informatics from a rich legacy of networks and conceptual resources. Information Technology \& People 18: 9-20.

Laperrière, A. 1997. Les critères de scientificité des méthodes qualitatives. In La recherche qualitative : Enjeux épistémologiques et méthodologiques, eds. Groupe de recherche interdisciplinaire sur les méthodes qualitatives and J. Poupart. Montréal.

Lavoie, B., G. Henry, and L. Dempsey. 2006. A service framework for libraries. D-Lib Magazine 12.

Lincoln, Y.S. \& E.G. Guba. Naturalistic inquiry. 1985. Beverly Hills, Californie, Sage.

Lynch, C.A. and J.K. Lippincott. 2005. Institutional Repository Deployment in the United States as of Early 2005. 2006. Available from http://www.dlib.org/dlib/september05/lynch/09lynch.html [April 2007].

Markus, M.L. and D. Robey. 1983. The Organizational Validity of Management Information-Systems. Human Relations.

Martineau, S. 2005. L'observation en situation : enjeux, pratiques et limites. Paper presented at L'instrumentation dans la collecte des données, 24 2006, TroisRivières (UQTR).

Miles, M.B. and A.M. Huberman. 2003. Analyse des données qualitatives. Paris : De Boeck Université.

Orlikowski, W.J. 1992. The Duality of Technology - Rethinking the Concept of Technology in Organizations. Organization Science.

Orlikowski, W.J. and J.J. Baroudi. 1991. Studying Information Technology in Organizations : Research Approaches and Assumptions. Information Systems Research 2: 1-28.

Patton, M.Q. 2002. Qualitative research \& evaluation methods. Thousand Oaks, Calif. : Sage Publications.

Salaün, J.M. 2004. Libre accès aux ressources scientifiques et place des bibliothèques. Bulletin des bibliothèques de France 49: 20-30. 
-----. 2006. Économie du document - Pour des archithécaires. In Pérenniser le document numérique, eds. Calderan, L., B. Hidoine, and J. Millet ADBS-Édition.

Sawyer, S. and K.R. Eschenfelder. 2002. Social informatics: Perspectives, examples, and trends. Annual Review of Information Science and Technology 36: 427-65.

Sparks, S. JISC disciplinary differences report. 2005. Londre, Rightscom. Available from http://www.jisc.org.uk/uploaded_documents/Disciplinary\%20Differences\% 20and\%20Needs.doc [April 2007].

Suber, P. 2005. Open access, impact, and demand - Why some authors self archive their articles. British Medical JournaL 330: 1097-98.

Swan, A., P. Needham, S. Probets, A. Muir, C. Oppenheim, A. O'Brien, R. Hardy, F. Rowland, and S. Brown. 2005. Developing a model for e-prints and open access journal content in UK further and higher education. Learned Publishing.

Walsham, G. 1993. Interpreting information systems in organizations. Chichester: Wiley.

-----. 1995. Interpretive Case-Studies in Is Research - Nature and Method. European Journal of Information Systems 4: 74-81.

-----. 2006. Doing interpretive research. European Journal of Information Systems 15: 320-330.

Xie, H. and D. Wolfram. 2002. State digital library usability : Contributing organizational factors. Journal of the American Society for Information Science and Technology 53: $1085-97$.

Yin, R.K. 2003. Case study research : design and methods. Thousand Oaks, Calif. : Sage Publications.

\section{Note}

${ }^{1}$ On consultera à ce sujet l'excellente bibliographie de Charles Bailey disponible en ligne à l'adresse suivante : http://epress.lib.uh.edu/sepb/sepb.html [April 2007]. 